\title{
Testing the transmitted wavefront of large aperture long-focal- length lens using a multizone computer-generated hologram
}

\author{
Jian-Peng Cui", Ning Zhang, Jie Liu, Di-Long Wu, Hua Xu, Ding-yao Yan and Ping Ma \\ Chengdu Fine Optical Engineering Research Center, 610041 Chengdu, China
}

\begin{abstract}
A method for testing the transmitted wavefront of large aperture long-focal-length lens with a multizone computer-generated hologram $(\mathrm{CGH})$ is proposed. The multizone $\mathrm{CGH}$ has 5 zones: one main zone for the null testing of long-focal-length lens and four auxiliary zones for the pre-alignment of measured lens. Both $1^{\text {st }}$ order wavefront and $0^{\text {th }}$ order wavefront of CGH are measured, and $0^{\text {th }}$ order wavefront is used to calibrate the substrate error. To verify this test approach, a $450 \mathrm{~mm} \times 450 \mathrm{~mm}$ multizone CGH is designed and fabricated for testing the spatial filter lens. Experiments and error analysis are carried out. The results show that the desired precision can be reached with use of $\mathrm{CGH}$.
\end{abstract}

\section{Introduction}

Large aperture long-focal-length lens has been widely used as the spatial filter lens in high power laser system, such as National Ignition Facility in Unite States, Laser Mégajoule in France, Gekko XII in Japan. Transmitted wavefront is an important specification for long-focallength lens and will affect the filtering effect and beam quality directly. One method for testing the transmitted wavefront involves using a large convex mirror [1-2]. This method has a simple test configuration and short optical path. However, measurements of large convex mirrors are notoriously difficult because they require flat interferometer and auxiliary optics that are larger than the surfaces being tested, which results in a high cost.

Current developments in diffractive optics and lithography technology make the use of large aperture computer-generated hologram (CGH) an attractive alternative [3-4]. Therefore, in this paper, we propose to measure the transmitted wavefront of long-focal-length lens by using a reflective multizone $\mathrm{CGH}$. Compared with the convex mirror, CGH has a large advantage in cost, because it is cheaper to manufacture a diffractive element than convex mirrors of equivalent size. Moreover, the design of $\mathrm{CGH}$ is very flexible, one multizone CGH can provide the wavefront correction for null testing and prealignment of the lens under test simultaneously, which makes the alignment of optical elements easier.

\section{Measurement principle}

Figure 1 shows the CGH test configuration for longfocal-length lens. A large aperture Fizeau interferometer and a reflective multizone $\mathrm{CGH}$ is used to measure the transmitted wavefront. The multizone $\mathrm{CGH}$ consists of one main zone and four auxiliary zones. The main zone is located at the center of the substrate. In this zone, testing
CGH with circular lines is etched to emulate, in $1^{\text {st }}$ order (or higher order), the reflective properties of the convex mirror. Auxiliary zones are four square zones and located around the main zone. In auxiliary zones, four beam projection CGHs are etched for the prealignment of the measured lens by projecting four marks around the edges of the measured lens.

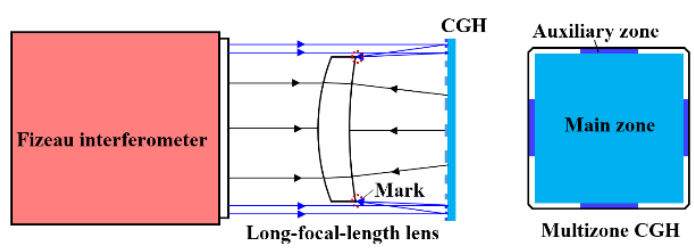

Fig. 1. Schematic drawing of the CGH test configuration for long-focal-length lens.

As we know, CGH substrate figure error is usually the primary error source in the $\mathrm{CGH}$ measurement. To calibrate the $\mathrm{CGH}$ substrate figure error, two measurements are made: $0^{\text {th }}$ order measurement and $1^{\text {st }}$ order measurement. Through measuring the $0^{\text {th }}$ order diffraction wavefront of the $\mathrm{CGH}$, we can obtain the substrate figure error and back it out from $1^{\text {st }}$ order one.

\section{Experiments and results}

\section{1. $0^{\text {th }}$ and $1^{\text {st }}$ order measurements}

To verify the feasibility of this test approach, we designed a multizone $\mathrm{CGH}$ for testing the spatial filter lens. This spatial filter lens has a size of $430 \mathrm{~mm} \times 430 \mathrm{~mm}$ and thickness of $46.5 \mathrm{~mm}$. The effective focal length $f$ is $31250 \mathrm{~mm}$ (@ $\lambda=1053 \mathrm{~nm})$ and the vertex radii of curvature are $\mathrm{R} 1=9377.868 \mathrm{~mm}(\mathrm{CX})$ and $\mathrm{R} 2=28133.886 \mathrm{~mm}(\mathrm{CC})$, respectively. In $410 \mathrm{~mm} \times 410 \mathrm{~mm}$ clear aperture, the requirement of transmitted wavefront is $\mathrm{PV}<\lambda / 3$

\footnotetext{
* Corresponding author: 547150445@qq.com
} 
(@ $\lambda=632.8 \mathrm{~nm}$ ). The multizone CGH is fabricated on a $450 \mathrm{~mm} \times 450 \mathrm{~mm} \times 70 \mathrm{~mm}$ fused silica substrate and the substrate is specified to have a figure of $P V \sim 1 / 10 \lambda$. The testing CGH is $430 \mathrm{~mm} \times 430 \mathrm{~mm}$ and the minimum line spacing is $32.1 \mu \mathrm{m}$. In our experiment setup, the large aperture interferometer is a commercialized Fizeau interferometer INF600-LP produced by Tyggo, which has a test aperture of $610 \mathrm{~mm}$, as shown in Fig. 2.
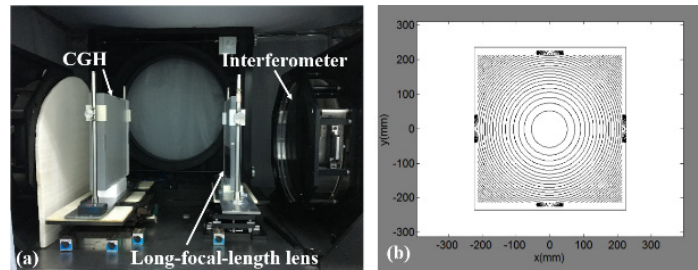

Fig. 2. (a) The experiment setup for testing the long focus lens with $\mathrm{CGH}$; (b) the fringe patterns of multizone $\mathrm{CGH}$.

Figure 3 shows two measurements for $0^{\text {th }}$ and $1^{\text {st }}$ order. We can see that there are ghost fringes near the center of the lens. These ghost fringes are caused by the reflections of lens surface and the diameter are $1.38 \mathrm{~mm}$ and $49.76 \mathrm{~mm}$ for the first and second surface, respectively. To calibrate CGH substrate, the $0^{\text {th }}$ order map is transformed to match $1^{\text {st }}$ order map. Figure 4 shows the mapping function between the radius positions of $\mathrm{CGH}$ and the measured lens. After CGH substrate calibration, the final transmitted wavefront with $\mathrm{CGH}$ substrate calibrated is $\mathrm{PV}=0.2284 \lambda$ and $\mathrm{RMS}=0.0276 \lambda$.

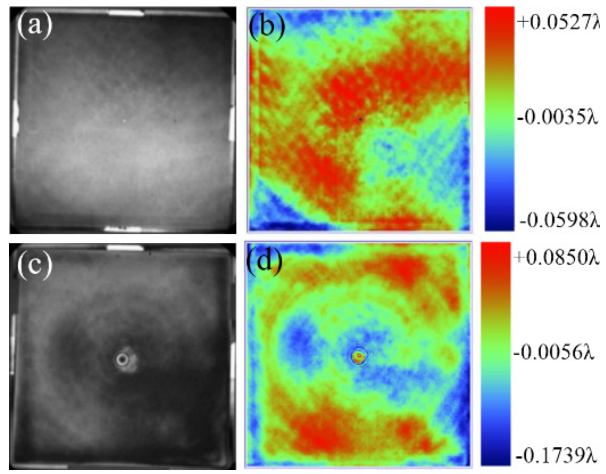

Fig. 3. $0^{\text {th }}$ and $1^{\text {st }}$ order measurements. (a) Interferogram for $0^{\text {th }}$ order; (b) CGH substrate figure map: $\mathrm{PV}=0.1125 \lambda$ and $\mathrm{RMS}=0.0178 \lambda$; (c) Interferogram for $1^{\text {st }}$ order; (d) Wavefront map: $\mathrm{PV}=0.2590 \lambda$ and $\mathrm{RMS}=0.0304 \lambda$.
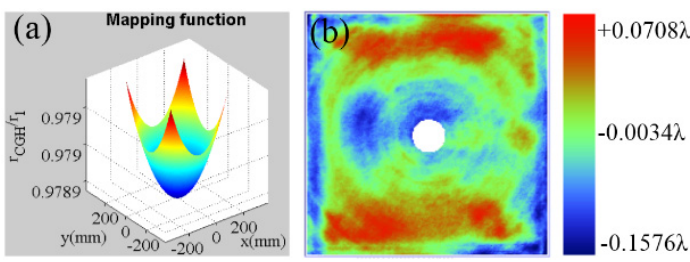

Fig. 4. Data mapping between $0^{\text {th }}$ order and $1^{\text {st }}$ order. (a) Mapping function; (b) Wavefront map with CGH substrate calibrated: $\mathrm{PV}=0.2284 \lambda$ and $\mathrm{RMS}=0.0276 \lambda$.

\subsection{Error analysis}

The CGH test errors includes design error, fabrication errors, alignment errors, and mapping errors, as given in Table 1. Assuming all the errors are un-related and independent, the total wavefront error of this CGH test can be estimated as the root-sum-spuare (RSS) of these errors, which is approximately 0.0091 $\lambda$ RMS. Because the test beam passes through the measured lens twice, the final transmitted wavefront error for long-focal-length lens is half of the RSS value, which is $0.00455 \lambda$ RMS.

Table 1. Wavefront errors analysis for the CGH test

\begin{tabular}{|c|c|c|}
\hline \multicolumn{2}{|c|}{ Source of Errors } & $\begin{array}{l}\text { Wavefront } \\
\text { error }\end{array}$ \\
\hline $\begin{array}{l}\text { Design error } \\
\text { of CGH }\end{array}$ & Design residual & $0.0000 \lambda$ \\
\hline \multirow{4}{*}{$\begin{array}{l}\text { Fabrication } \\
\text { errors of } \mathrm{CGH}\end{array}$} & $\begin{array}{l}\text { Pattern distortion error } \\
(0.5 \mu \mathrm{m})\end{array}$ & $0.0051 \lambda$ \\
\hline & Etching depth error $(5 \mathrm{~nm})$ & $0.0000 \lambda$ \\
\hline & Duty-cycle error $(5 \%)$ & $0.0070 \lambda$ \\
\hline & $\begin{array}{l}\text { Substrate figure calibration } \\
\text { residual }(0.0020 \lambda)\end{array}$ & $0.0020 \lambda$ \\
\hline \multirow{2}{*}{$\begin{array}{c}\text { Alignment } \\
\text { errors of } \mathrm{CGH}\end{array}$} & $x$ tilt (1 fringe) & $0.0000 \lambda$ \\
\hline & $y$ tilt (1 fringe) & $0.0000 \lambda$ \\
\hline \multirow{2}{*}{$\begin{array}{c}\text { Fabrication } \\
\text { errors of } \\
\text { measured lens }\end{array}$} & Radius of curvature $(0.1 \%)$ & $0.0003 \lambda$ \\
\hline & Thickness of lens $(0.2 \mathrm{~mm})$ & $0.0000 \lambda$ \\
\hline \multirow{5}{*}{$\begin{array}{c}\text { Alignment } \\
\text { errors of } \\
\text { measured lens }\end{array}$} & $x$ decenter $(0.2 \mathrm{~mm})$ & $0.0002 \lambda$ \\
\hline & $y$ decenter $(0.2 \mathrm{~mm})$ & $0.0002 \lambda$ \\
\hline & $x$ tilt $\left(0.04^{\prime}\right)$ & $0.0002 \lambda$ \\
\hline & $y$ tilt $\left(0.04^{\prime}\right)$ & $0.0002 \lambda$ \\
\hline & $z$ displacement $(1 \mathrm{~mm})$ & $0.0000 \lambda$ \\
\hline \multirow{2}{*}{$\begin{array}{l}\text { Mapping } \\
\text { errors }\end{array}$} & $x$ direction (1 pixel) & $0.0012 \lambda$ \\
\hline & $y$ direction (1 pixel) & $0.0012 \lambda$ \\
\hline \multicolumn{2}{|r|}{ RSS Errors } & $0.0091 \lambda$ \\
\hline
\end{tabular}

\section{Conclusion}

A method for measuring the transmitted wavefront of long-focal-length lens with a reflective multizone $\mathrm{CGH}$ is proposed. In this method, a reflective $\mathrm{CGH}$ with circular lines is employed as an alternative of the convex mirror. This avoids the manufacture of large aperture convex mirror and keeps the advantage of simple test configuration. Four beam projection CGHs are designed to help the pre-alignment of the measured lens, which significantly improves the testing efficiency. Experiments and error analysis exhibit that this multizone $\mathrm{CGH}$ approach has a good performance.

\section{References}

1. C. Jin, S. Liu, Y. Zhou, X. Xu, C. Wei, J. Shao, Chin. Opt. Lett. 12, S21203 (2014).

2. T. G. Parham, T. J. McCarville, and M. A. Johnson, Optical Fabrication and Testing, OWD8, (2002).

3. J. C. Wyant, V. B. Bennett, Appl. Opt. 11, 2833, (1972).

4. B. DeBoo, J. Sasian, Appl. Opt. 42, 3903 (2003). 